\title{
Experiencia, pragmatismo y líneas de actuación comunes, bases del nuevo modelo de desarrollo rural del País Vasco
}

\section{Experience, pragmatism and common lines of action, bases of the new model of rural development of the Basque Country}

\author{
Juan Cruz Alberdi Collantes*
}

A finales de los noventa la Comunidad Autónoma pone en marcha una nueva iniciativa de desarrollo rural fundamentada no sólo en los objetivos, programas y presupuestos de la Comunidad Europea sino en las posibilidades que la orientación y la coordinación de las administraciones públicas del País Vasco pueden aportar al desarrollo rural. La Ley de Desarrollo Rural, aprobada en Abril de 1998, señala que las políticas orientadas de manera específica al desarrollo de las zonas rurales del País Vasco adoptarán la forma de programas de desarrollo rural. En la elaboración de estos programas se elige la comarca como ámbito preferente de actuación.

Con la aprobación de la Ley se realizaron los primeros Planes de Desarrollo Rural (PDR) de las comarcas del País Vasco. Se caracterizaron por ser unos documentos participativos, apoyados con la recopilación de abundante información estadística, abiertos a buena parte de la sociedad rural y que crearon ilusión entre los participantes y agentes de desarrollo rural. El documento, sin embargo, careció del anunciado apoyo de la administración y dejo entrever la debilidad de un modelo fundamentado en la buena voluntad de las diferentes administraciones.

\footnotetext{
* Departamento de Geografía de la Universidad del País Vasco (juancruz.alberdi@ehu.es).
} 
Una década después asistimos a la actualización de sus contenidos, ejercicio de reflexión que está dejando en evidencia la necesidad de plantear un nuevo modelo de actuación en materia de desarrollo rural, con una metodología que limite la participación, más centralizada y direccionalista. Los objetivos perseguidos en los nuevos Planes Comarcales son menos pretenciosos, más realistas y apoyados en las posibilidades reales que tienen los agentes de desarrollo.

En el trabajo presentamos un modelo de organización y financiación dirigido al progreso de los espacios rurales, apoyado preferentemente en los recursos locales y regionales, una alternativa a aquellos programas sustentados en la iniciativa y presupuestos comunitarios, un modo de organización compartido por muchas regiones que apenas cuentan con recursos europeos y que, previsiblemente, se planteará en los próximos años en muchas regiones del sur de Europa.

\section{La Ley de Desarrollo Rural, una herRAmienta ADECUADA POR DESARROllar}

La Ley de Desarrollo Rural (1998) supone el reconocimiento oficial del carácter multifuncional del espacio rural vasco, con un habitante que genera riqueza pero que también gestiona territorio y protege y regula el espacio en el que vive. Pretende definir los objetivos que deben orientar las actuaciones de las administraciones públicas en materia de desarrollo rural, propone el establecimiento del marco normativo en el que se han de desarrollar las políticas orientadas de manera específica al desarrollo de las zonas rurales y determina los instrumentos que permitan adecuar el resto de políticas y actuaciones administrativas con la consecución de los objetivos del desarrollo rural que se establecen en la Ley ${ }^{1}$.

El modelo organizativo que propone la Ley de Desarrollo Rural se apoya en la necesidad de que las políticas orientadas de manera específica al desarrollo

\footnotetext{
${ }^{1}$ A diferencia de las políticas que se sustentan en la participación exterior, como el Leader o el Proder. Por ejemplo, como señala Plaza (2005), el coste total de la inversión de PRODER I fue de 619,2 millones de euros; de ellos la mayor parte, el 45,2\% (279,7 millones de $€$ ), eran de financiación comunitaria (a través de los fondos Feder y Feoga-Orientación), poco más de la tercera parte $(34,7 \%)$ provenían de financiación privada, de agentes privados (214,15 millones de $€$ ) y la quinta parte restante 20,1\%, 125 millones de $€$ ) los aportaban las distintas administraciones (la estatal, representada en el MAPA, con el 2,6\%: 16,0 millones; la autonómica, con el 9,5\%: 59,0 millones de $€$; y la local: 8,0\%, 49,9 millones de €).
} 
de las zonas rurales del País Vasco adopten la forma de programas de desarrollo rural. En la elaboración de estos programas se elige la comarca como ámbito preferente de actuación, por la convicción de que el nivel comarcal se muestra como el más adecuado para emprender tal labor puesto que, habitualmente, se trata de un espacio cohesionado, con una relación funcional manifiesta y con una problemática socioeconómica similar.

Entre la asunción de competencias cabe resaltar la necesidad de que, tanto el Gobierno Vasco como las diputaciones y ayuntamientos, han de participar en la elaboración de los Planes de Desarrollo Rural puesto que, su aprobación, lleva implícita una obligatoriedad de aceptación y de ejecución en el ámbito de competencia de cada institución.

Las labores de coordinación y colaboración entre las distintas instituciones la ejerce Landaberri, en cuyo seno se produce la cooperación y colaboración necesarias para la aplicación de la presente ley. Está adscrito al Departamento del Gobierno Vasco recayendo, la presidencia y la responsabilidad de coordinación, en el área de Agricultura y Desarrollo Rural. Está formada por representantes del Gobierno Vasco, las Diputaciones Forales y los municipios del País Vasco

Si Landaberri es el órgano que ha de cumplir la función de coordinar y dirigir las actuaciones en materia de desarrollo rural, la aplicación de los programas diseñados en cada comarca es realizada por órganos locales. En este sentido, se apuesta por la creación de una nueva figura, las Asociaciones de Desarrollo Rural (ADR) que, se establecen, como instrumento de participación y colaboración de los agentes económicos y sociales en las actuaciones de desarrollo rural. La Ley establece los requisitos para el reconocimiento de las ADR con el carácter de entidades de derecho privado y representativas, de los diferentes sectores económicos y sociales de la zona comprendida en un PDR². Las

2 Cataluña, siguiendo las aportaciones que realiza Cáceres (1996) pone en marcha desde 1989 lo que denominan Planes Comarcales de Montaña, con dos funciones básicas: coordinar todas las actividades e iniciativas públicas y tener una misión económica y social del desarrollo. Se regulan por consejos comarcales e incluso se coordinan por un Consejo General de Montaña. A su vez, se fundamentan en planes comarcales, en este caso de tipo quinquenal, fundamentados en un estudio socio-económico previo: la Dirección General de Planificación y Acción Territorial. Los objetivos e incluso el funcionamiento es similar al que el Gobierno Vasco aprueba en 1998, salvo el enfoque más participativo y local que quiere aportar esta última Comunidad y, la concreción más inmediata en un documento elaborado por los propios agentes comarcales y en su plasmación en planes de actuación y seguimiento anual. No ocurría así en la coordinación de las iniciativas del programa Leader, que eran habitualmente diseñadas y dirigidas por la Consejería de Agricultura de cada Comunidad (ver Corbera (1999) en el caso de Cantabria; Moreno (1996) en la serranía de Cuenca, etc.). 
ADR vienen a dar continuidad a la labor de los grupos de acción local creados a raíz de la Ley de Agricultura de Montaña de 1986 y que, en la Comunidad Autónoma Vasca, participaban en la gestión del programa Leader y 5B.

El mismo año en que es aprobada la Ley de Desarrollo Rural, Landaberri encarga la elaboración de los programas de desarrollo rural, de aquellos que coinciden con las comarcas que en ese momento están clasificadas como zonas 5B y que, se benefician de los programas de la iniciativa Leader II y, dos años después, del resto de las comarcas del País Vasco (ver figura 1).

FIGURA 1

PLANES DE DESARROLLO RURAL INICIADOS EN 1998 EN EL PAÍS VASCO

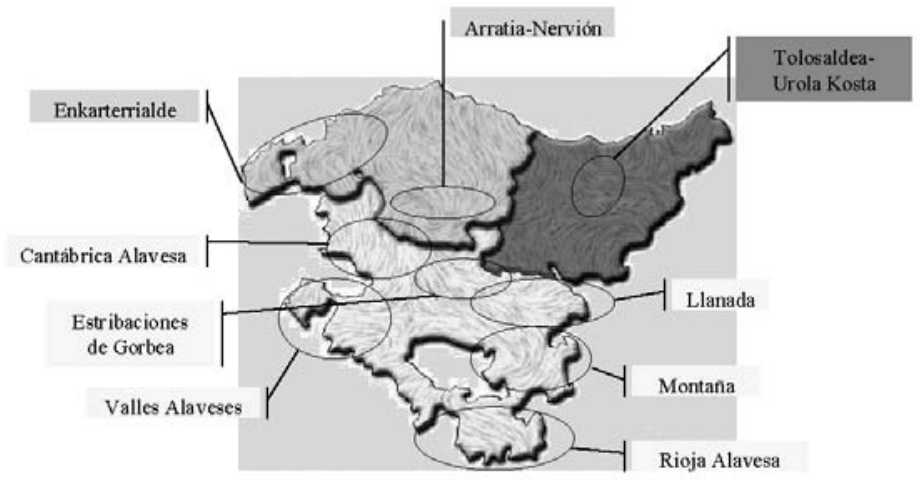

PLANES DE DESARROLLO RURAL INICIADOS EN 2001 EN EL PAÍS VASCO

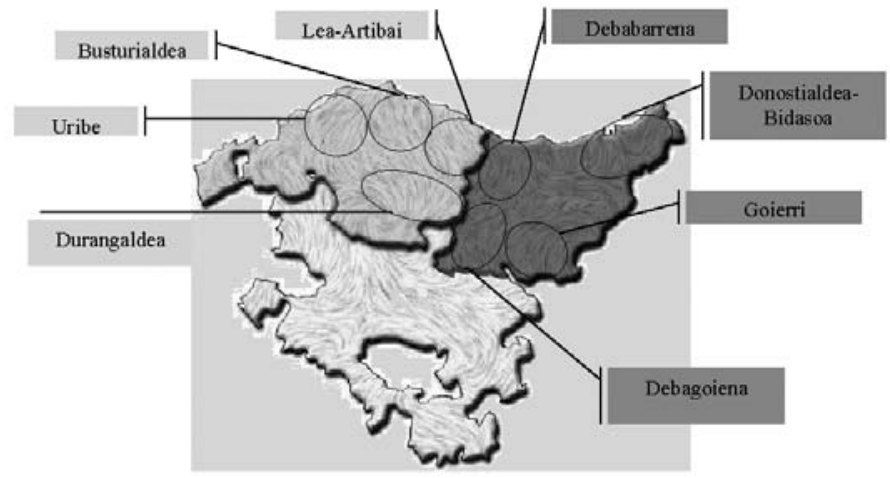

Fuente: Centro de Desarrollo Rural Mendikoi. Elaboración propia. Año 2003.

Estudios Geográficos, Vol. LXXI, 268, pp. 7-38, enero-junio 2010

ISSN: 0014-1496, eISSN: 1988-8546, doi: 10.3989/estgeogr.0433 
Teniendo en cuenta que se trata de un programa integral, realizado bajo la consideración de que el desarrollo rural requiere de actuaciones que incidan sobre los distintos aspectos que condicionan la actividad y la calidad de vida en el medio rural (actividad económica, vivienda, equipamientos y servicios, infraestructuras, medio ambiente, etc...), a lo largo del proceso se ha promovido la participación de agentes representativos de los distintos ámbitos objeto de análisis. Por todo ello, se ha definido un sistema de participación basado en la configuración de diversos grupos de trabajo, capaces de aportar las distintas visiones que hacen posible un diseño completo del desarrollo del medio rural de la comarca. Son unos Planes participativos, abiertos al conjunto de los habitantes del medio rural, y ambiciosos, en la medida en que pretenden abarcar todos aquellos aspectos que condicionan el progreso de este medio.

En términos generales podemos señalar que la elaboración y ejecución de los Planes de Desarrollo Comarcales han permitido conocer e impulsar la reflexión sobre la situación del medio rural y, en la medida que se han ido materializando proyectos, contribuir al desarrollo de este espacio.

Sin embargo, a pesar de que algunas de las demandas y propuestas que se planteaban en los planes de desarrollo rural están siendo solventadas en los programas que se han iniciado, las perspectivas generadas a raíz de la aprobación de la Ley de Desarrollo Rural en términos generales no se han cumplido.

En las reflexiones efectuadas por los agentes rurales ${ }^{3}$ el aspecto más criticado es la falta de compromiso económico adquirido que respalde los PDR 4 . Es más, según ellos, la política desarrollada no responde a las prioridades establecidas en los planes de desarrollo rural.

Se tiene la convicción de que los PDR, no han sido asumidos como una herramienta de utilidad cotidiana. Se observa que, ni la administración ni los

\footnotetext{
${ }^{3}$ Los resultados de las jornadas se recogen en un informe interno titulado «Ley de Desarrollo Rural y Programas de Desarrollo Rural. Jornadas de reflexión 14, 15, 18 y 24 de Julio de 2003», organizadas por Mendikoi y el área de Desarrollo Rural del Gobierno Vasco.

${ }^{4}$ Lo que no quiere decir que sean las ADR las que materialicen los programas, a diferencia de iniciativas como el Proder, donde son los GAL los que la ejecutan. A este respecto Plaza (2005) señala que la diferenciación entre Comunidades Autónomas de uno y otro tipo influye, igualmente, en los «gestores-beneficiarios» de las ayudas recibidas, puesto que éstos serán, bien los Grupos de Acción Local, cuando la Comunidad Autónoma decide que la gestión de los programas se realice mediante este método organizativo o bien las propias Comunidades Autónomas (como en el caso de Aragón, Cataluña y Madrid por ser regiones fuera de Objetivo 1), o las entidades locales cuando, cualquiera que sea la modalidad de financiación, las ayudas a proyectos de perceptores finales sean concedidas, a propuesta de Grupos de Acción Local por Administraciones locales, Sociedades de Promoción o Comunidades Autónomas.
} 
agentes sociales dan a la Ley y a los PDR la importancia que realmente tienen o pueden tener. En este sentido, se apunta a una falta de consenso político, a pesar de que fue aprobada por unanimidad, falta de credibilidad en lo que señala tanto la Ley como los PDR, inexistencia de coordinación entre los distintos niveles de la administración... En definitiva, una falta de interiorización de la importancia que posee llevar adelante el desarrollo del medio rural y con ello, un desánimo generalizado entre entidades locales y Asociaciones de Desarrollo Rural, que se sienten protagonistas débiles en el cumplimiento de la función para la que, al menos estas últimas, fueron creadas.

Los agentes rurales consideran a la Ley de Desarrollo Rural como una herramienta adecuada y necesaria para emprender una política real de promoción rural. Su materialización, sin embargo, depende de la voluntad y recursos de las diferentes administraciones y es aquí donde apenas se ha avanzado. Los contenidos de la Ley, por tanto, son reiteradamente ignorados hasta el punto de que, muchas de las estructuras más básicas ni han iniciado su andadura.

La necesidad de renovar aquellos programas realizados hace una década, lleva a plantearse unos objetivos diferentes a los que inicialmente se recogían. Las demandas de la población local no han variado pero ha cambiado la mentalidad de las ADR, más centrada en sus posibilidades que en ser estandarte de demandas difíciles de satisfacer. En la redacción de los nuevos documentos cambia la metodología, más ligera y dirigida, y cambian los contenidos, más realistas y alcanzables.

\section{HACIA UNA METODOLOGÍA DE DESARROLLO RURAL AJUSTADA A LAS POSIBILIDADES DE MATERIALIZACIÓN}

La revisión de los Planes de Desarrollo Rural requiere, como primer paso, someter a crítica la metodología que se utilizó en la elaboración, puesta en marcha y seguimiento de los anteriores. Aquellos planes pecaron de exceso de información y de pretenciosidad, convirtiéndose en herramientas de escasa utilidad para los agentes rurales, habitantes del medio rural y para las entidades públicas implicadas en el desarrollo rural.

Los contenidos de la metodología a aplicar son presentados por un grupo de trabajo compuesto por diferentes investigadores de la Universidad del País Vasco, la consultora LKS e Itsasmendikoi y son sometidos a discusión en diferentes encuentros celebrados durante el 2007 con los agentes de las diferentes Asociaciones de Desarrollo Rural. 


\section{Pragmatismo y sencillez, entre los caracteres de la nueva propuesta metodológica}

La propuesta metodológica, en principio, pretende restar oficialidad, participación e incluso estudio del medio para ganar en pragmatismo y concreción. Se busca la practicidad de las acciones, la implicación administrativa a partir de una dirección visible a ejercer por el Departamento de Agricultura, la obtención de fuentes de financiación que aporten capacidad de ejecución a las propuestas presentadas y todo ello persiguiendo un modelo de actuación realista y medible. Concretamos en cuatro las premisas que han de regir la elaboración de los nuevos Planes de Desarrollo Rural:

- Metodología más operativa, sencilla y práctica. Se pretende pasar de un plan extenso y descriptivo, dominado por el diagnóstico, a otro centrado en los aspectos estratégicos. Se quiere un programa abarcable y manejable.

- Programas de desarrollo realistas y de aplicabilidad. Se propone pasar del desideratum que supuso el anterior a unos planteamientos más posibilistas. La importancia del Plan no está en el nivel de conocimiento que sobre el medio aporte a unos agentes que llevan varias décadas trabajando en él, sino en la materialización de aquello que en dicho documento se ha recogido.

- Programas con operatividad y dinamismo. Se observa una necesidad de dotar de contenido práctico a unos Planes que aportaron estudio y conocimiento de área para lo que se hace fundamental hacer que estos documentos se conviertan en instrumentos de gestión, para lo que ha de dotárseles de herramientas financieras adecuadas.

- Mayor protagonismo y liderazgo de las Asociaciones de Desarrollo Rural. A pesar del protagonismo que les aportaba la Ley, en el anterior Plan han cumplido en muchos casos el papel de participante, o incluso de mero espectador. Frente a éste se reclama ahora el de líder que cumple la función de impulsar y gestionar el proceso.

Aparentemente, el esquema metodológico de los nuevos PDR no cambia respecto a los de la fase anterior. La estructuración es similar, coincidiendo con la utilizada habitualmente en la redacción de planes de desarrollo y que se concreta en una primera fase de organización del proceso, un diagnóstico, formulación de la estrategia, planificación para finalizar en una última fase de despliegue, seguimiento y actualización.

Una vez examinados los planteamientos realizados en cada uno de los aportados ya se observa una evolución importante respecto a la metodología anterior e incluso a la utilizada habitualmente en la redacción de planes de de- 
sarrollo. La década trascurrida, junto a la inoperancia de los anteriores planes, ha hecho reflexionar a los actores que intervienen en el desarrollo rural, ganando en pragmatismo y utilidad aunque tal vez sea a cuenta de perder en participación e información 5 .

El análisis de cada uno de los diferentes apartados deja en evidencia las diferencias establecidas respecto al anterior documento y vamos observando como estos planes ganan en sencillez y agilidad, operatividad y factibilidad, protagonismo y autonomía creciente de las ADR, mayor componente estratégico y carácter selectivo de las propuestas, en utilidad y engarce de los PDR con el diseño de políticas y programas por parte de las distintas administraciones e incluso en el énfasis que se pone en la evaluación y actualización permanente.

\section{El proceso metodológico mantiene sus fases clásicas pero los contenidos de cada una son alterados}

El nuevo modo de proceder se evidencia desde el primer apartado en el que se distribuyen los planes de desarrollo rural, la organización del proceso. Entre las que destacamos cabe señalar las siguientes:

- En toda la organización del proceso se establece un responsable máximo, el gerente de la ADR correspondiente. A él compete tomar las decisiones prioritarias (quién y cómo participa, a quién se entrevista, qué información se analiza,...). El gerente ha de consultar a la junta de la ADR, cuenta con el asesoramiento de ItsasMendikoi pero asume ahora la responsabilidad de que la organización sea correcta, el diagnóstico adecuado, se formulen las estrategias ajustadas y se planifique su materialización y seguimiento. La justificación de esta decisión estriba en la necesidad de crear un plan ágil y sencillo para unas asociaciones en las que a menudo el gerente es el motor de la misma. La importancia de la ADR en la elaboración, ejecución y seguimiento de los programas ya se establecía en los anteriores planes aun-

\footnotetext{
5 Observamos que en materia de desarrollo rural cada vez más se tiende a buscar unos procedimientos de actuación que impliquen al conjunto de las administraciones. Como consecuencia de las propuestas de evaluación de los programas de desarrollo rural que se están sucediendo en toda Europa, cada vez son más las regiones que tratan de aplicar unos procedimientos más directos, completos y, sobre todo, que busquen las implicaciones del conjunto de administraciones. Buen ejemplo de ello son las políticas de evaluación de proyectos de desarrollo rural aplicadas en el Reino Unido y recogidas bajo la denominada «Metodología de Cardiff» (Bristol, G. et al., 2001).
} 
que siempre era una acción compartida, con un ámbito de decisión que le supera y una responsabilidad que en todo caso recaía en la Junta. Ahora no sólo es la ADR la principal receptora sino que, es el gerente el que en gran medida va a marcar la estrategia a seguir.

- El modo de participación cambia sustancialmente. De apostar por un modelo abierto, apoyado en diferentes grupos de trabajo y en dinámicas de encuentros sectoriales y comarcales, se prima la participación selectiva, dirigida por un objetivo, sustentada en la entrevista y promovido por la gerencia de la ADR. Con ello se trata de reducir el exceso de información y aportaciones que, en la anterior fase, restaron operatividad al documento presentado 6 .

- Las fuentes de información, al igual que el modelo de participación, se reducen considerablemente. En términos generales apenas se utilizan datos estadísticos y se opta por tomar como referente la opinión que, en función de los objetivos planteados, han ido aportando las diferentes personas entrevistadas. Con ello se consigue un documento abarcable y manejable, frente al carácter de volumen que tenía el anterior ${ }^{7}$.

La organización propuesta condiciona el carácter del proyecto. El objetivo del diagnóstico es analizar la situación del medio rural de la comarca. Sin embargo, es habitual pecar de excesiva exhaustividad, acumulación de datos y opiniones, y se convierte en una mera recopilación descriptiva de multitud de variables. De hecho, casi todo son problemas y limitaciones y todo parece te-

${ }^{6}$ Los grupos de discusión, por ejemplo, pierden importancia, con lo que se pierde una herramienta metodológica importante. En los grupos de discusión, como señala Viñas (2004), los miembros del grupo generan nuevas ideas porque se estimulan entre ellos. La dinámica del grupo empuja a los participantes a responder a las opiniones, comentarios e ideas de los otros, surgiendo nuevas líneas o perspectivas de análisis, que no hubieran sido identificadas con una encuesta estructurada. Las entrevistas abiertas o semi-estructuradas, los grupos de discusión, las reuniones de comunidad, los estudios de caso, la observación participante, etc., son técnicas cualitativas de recogida de información. Todas ellas permiten recoger de forma más acertada la información que no había sido previamente pensada por la persona encuestada, ya que ésta no se encuentra forzada a elegir una, y sólo una, de las respuestas predeterminadas, gozando de más libertad de expresión.

${ }^{7}$ Las posibilidades de éxito de esta fase estriban en tener la habilidad de crear un esquema de trabajo que pase de la planificación compartida a la acción compartida, para lo que se hace obligatorio concertar actuaciones con agentes claves. En definitiva, como señala Viñas (2004) en su análisis sobre la evolución cualitativa de los programas de desarrollo en las zonas rurales, se trata de poner en marcha un enfoque cualitativo fundamentado en una reflexión previa y que ayude a decidir en cada caso la combinación metodológica más adecuada. 
ner la misma importancia. Es más, la propia elaboración del diagnóstico ha acaparado habitualmente buena parte del protagonismo y esfuerzos. Para solventar esta cuestión se propone utilizar y sacar el máximo rendimiento de la experiencia y el conocimiento previo existente sobre la realidad del medio rural y sobre los efectos producidos por la aplicación de anteriores programas para delimitar los ámbitos y variables preferentes de análisis en cada comarca.

En este sentido, la actualización del diagnóstico debe realizarse a partir de un proceso más inductivo que deductivo ${ }^{8}$, evitando de este modo los errores más frecuentes en la elaboración del diagnóstico mencionados con anterioridad, así como el hecho de que se convierta en un estudio descriptivo, con tantas matrices DAFO como ámbitos de análisis, donde todos los elementos tienen la misma importancia y donde se pierden u ocultan los aspectos diferenciales de las distintas zonas rurales. La reflexión parte por tanto, del conocimiento y la experiencia adquirida en la etapa anterior y el diagnóstico ha de hacer referencia únicamente a aquellos ámbitos y variables que sean verdaderamente relevantes para el mantenimiento del medio rural de cada comarca y su desarrollo futuro ${ }^{9}$.

En la medida en que la actualización de los PDR se desarrolle en paralelo en todas las comarcas de la Comunidad Autónoma del País Vasco, la identificación de temas críticos comunes a todas las zonas o comarcas constituye una tarea que aporta valor añadido al proceso. La identificación de las limitaciones o problemas prioritarios que han de afrontar las zonas rurales de todo un Territorio o del conjunto de la Comunidad para su pervivencia y desarrollo futuro (temas críticos), constituye el primer paso para plantear soluciones conjuntas y, por lo tanto, con mayores visos de viabilidad, eficiencia y aceptación por parte de los organismos con competencias en la materia.

La Formulación de la Estrategia adquiere ahora un peso fundamental. El objetivo y resultado de esta fase, es el de obtener una visión de futuro para el medio rural de la comarca, compartida por los diversos agentes que operan en ella, e identificar los principales ejes en los que sustentar la estrategia de desa-

\footnotetext{
${ }^{8}$ En la metodología anterior se planteaba una recogida de información muy exhaustiva y homogénea para todas las comarcas intentando responder al esquema y guía de análisis establecido por la Ley. La recopilación de esta información constituía la primera tarea a realizar en la elaboración del diagnóstico. Para la revisión de los PDR y la elaboración del diagnóstico en esta nueva fase la recopilación de información es posterior a otra serie de actividades y tareas previas.

${ }^{9}$ En gran medida, se propone romper con el diagnóstico interactivo en el que quedan insinuadas las estrategias impulsoras de la participación de las redes locales en los proyectos da desarrollo, modelo generalmente impulsado en los programas de desarrollo local (López, A. et al., 2003). Frente a ello se propone un modelo centrado en la experiencia previa y en la dirección de la participación a aquellas personas que previamente puedan aportar algo.
} 
rrollo del medio rural comarcal que van a permitir el logro de la visión en el tiempo. Ello supone, en primer lugar, marcase un horizonte a medio-largo plazo sobre el medio rural que se pretende alcanzar y, posteriormente, identificar los cuatro o cinco ejes de desarrollo prioritarios para la comarca. Esta es una labor a realizar por la ADR, en todo caso contrastada con la visión de una serie de agentes clave y que, pretende romper con el listado inabarcable de estrategias y subestrategias que se recogían en la fase anterior. Se trata de priorizar y concretar y no de recoger sin gran criterio de valoración todas las aportaciones que se realizaban ${ }^{10}$.

Partiendo de los principales Ejes de Desarrollo, se inicia la Fase de Planificación con la definición de los Objetivos del medio rural de la comarca (qué queremos alcanzar). Las Estrategias son líneas de acción que marcan el camino a seguir para alcanzar los Objetivos vinculados a los Ejes (cómo lograremos los objetivos fijados). El modo de actuar responde al esquema general presentado. Por ejemplo, a la hora de marcar los objetivos se ha de priorizar que la ADR tenga capacidad de actuación sobre el mismo y las estrategias han de constituir orientaciones claras sobre la dirección o línea de trabajo considerada más idónea para alcanzarlos. La definición de objetivos queda en manos de la ADR que, los contrastara mediante reuniones individuales con agentes clave. Con la redacción de esta fase se pone fin al PDR.

La fase de despliegue y seguimiento del programa se concreta, al igual que la anterior, en la apuesta por la realización de planes anuales, que tienen la función de pasar de los objetivos anuales a su materialización en planes de actuación. Se pretende que tengan un seguimiento, que vayan marcados por una serie de indicadores, que sean aprobados anualmente por las juntas... La propuesta, en este caso, no es sino una continuación de la anterior, con la salvedad de que en la anterior fase éstos no se materializaron ${ }^{11}$.

10 Desde hace algunos años, se ha empezado a considerar importante realizar evaluaciones participativas, es decir, evaluaciones en las que los beneficiarios no son meramente fuente de datos, sino que participan también en el diseño, realización y análisis de los datos de la evaluación. Esto se ha conseguido en diversos grados, pero al menos se ha extendido la idea de que tales intentos darán más calidad a las evaluaciones y permitirán a los evaluadores sacar conclusiones más pertinentes (Casley y Kumar, 1990b, 125; Murria, 2002; Patton, 2002).

11 A diferencia del proceso anterior, en el que este apartado no fue materializado, se pretende ahora producir y medir lo que se realice. Como señalan Esparcia, J. y Nogueras, J. (2000), la importancia de el apartado de seguimiento y actualización es consecuencia de la convicción de que las políticas de evaluación son un instrumento de aprendizaje y mejora de la eficacia en la toma de decisiones. La evaluación es el instrumento que mejor permite conocer qué resultados se están obteniendo y, en su caso, introducir los mecanismos de corrección oportunos. 
La metodología presentada responde a los objetivos que se había propuesto, primando la sencillez y agilidad, el carácter dirigido que le aporta el gerente de la ADR y la pretensión realista que se le quiere dar, apostando por la obtención de unos objetivos abarcables con la actividad de las Asociaciones. También es destacable la necesidad de, partiendo del ámbito comarcal, responder a las demandas comunes a todas las zonas rurales, como modo de actuación y presión sobre la administración.

La materialización de los programas, sin embargo, reduce mucho la participación de la población y agentes del medio rural, las aportaciones se centran en las posibilidades que tienen las ADR de poner actuaciones en marcha, a menudo muy centradas en determinados ámbitos de actuación, con un plan muy dependiente de la visión de la junta de la ADR y, especialmente, de su gerente que, aunque aporte una visión cualificada, difícilmente dará cabida al conjunto de modos y visiones relativas al medio rural 12 .

Es difícil conjugar operatividad con participación, sencillez con representación y direccionalidad con apertura. A medida que el tiempo pasa y los planes avanzan se apuesta por la operatividad pero se corre el riesgo de pensar que todo lo conocemos. Se han de combinar ambas filosofías intentando retener las virtudes que unos y otros nos aportan.

Por lo demás, el modelo de actuación presentado responde a la evolución propia de un programa de desarrollo rural que se fundamenta cada vez más en la práctica que año tras año va adquiriendo y que queda en evidencia una vez que se realiza un ejercicio de valoración de sus resultados. ${ }^{13}$

12 Conclusión que rompe en cierta medida con los principios que rigen la política de desarrollo rural. Como señala Zapatero (1998), la «Declaración de Cork» (1996) y, de manera implícita la Agenda 2000 (1997), coinciden en la necesidad de potenciar una política de desarrollo rural duradero. El desarrollo rural integrado, supone la aplicación de un nuevo enfoque que pretende una línea integradora entre la ordenación del territorio, la planificación ambiental y el desarrollo rural, buscando un enfoque endógeno, territorial y ascendente.

${ }^{13}$ Los antiguos PDR se completaron siguiendo la metodología y las fases aquí señaladas. En el planteamiento de la revisión que se realiza se propone mantener las fases metodológicas, consideradas todas ellas como imprescindibles, pero se plantea remodelar muchos de los contenidos que en su elaboración eran tenidos en cuenta. Este proceso es consecuencia de la propia evolución de los programas de desarrollo rural europeos que, como señalan Wojan, TR. y Rupasingha A. (2001), tienden cada vez más a poner en marcha metodologías prácticas fundamentadas en la experiencia adquirida durante años. 
LAS COMARCAS VASCAS, OBJETO DE LA NUEVA APLICACIÓN METODOLÓGICA EN MATERIA DE DESARROLLO RURAL

Una vez definido el modo de actuación se da inicio al proceso de actualización de los Programas de Desarrollo Rural Comarcal del País Vasco, logrando obtener una fotografía de las zonas rurales de las comarcas en el momento actual, definiendo una visión a futuro de las mismas y diseñando una planificación estratégica para el período 2007-2013.

Además de actualizar los PDR de cada comarca, uno de los objetivos planteados desde el inicio era poder contar con la identificación de aspectos comunes que se repiten en las diferentes comarcas o territorios, que permitan hacer planteamientos conjuntos, a escala supracomarcal, territorial o autonómico. Ello ha sido posible debido a que el proceso se ha desarrollado en paralelo en las diversas comarcas, utilizando una misma metodología y creando una dinámica de trabajo conjunto.

La identificación de las limitaciones o problemas prioritarios que han de afrontar las zonas rurales de todo un Territorio para su pervivencia y desarrollo futuro (temas críticos), constituye el primer paso para plantear soluciones conjuntas y por lo tanto con mayores visos de viabilidad, eficiencia y aceptación por parte de los organismos con competencias en la materia.

De ahí que, como parte del proceso de actualización de los PDR, se plantee la identificación de los temas críticos comunes a partir de la información aportada por cada una de las comarcas. Por otro lado, si bien las ventajas competitivas deben ser diferenciales y específicas de la comarca, es de gran utilidad la identificación de aspectos positivos comunes (potencialidades y oportunidades) al medio rural de distintas comarcas de un territorio en la medida en que pueden constituir también el germen de posibles estrategias o proyectos conjuntos que contribuyan al mantenimiento y desarrollo del medio rural.

Todo este proceso debiera ser la base de posibles estrategias de «reconversión» que requieran el concurso de administraciones supracomarcales, con competencias en distintos ámbitos o sectores con fuerte impacto sobre la situación actual y futura del medio rural, así como de modificaciones y ajustes de las políticas sectoriales a las particularidades del medio rural.

En Álava y Vizcaya se ha partido de la elaboración comarcal de los PDR para, a partir de esta primera reflexión, consensuar en diferentes encuentros los temas comunes para el conjunto del territorio

En Guipúzcoa, se ha iniciado el trabajo de planificación a nivel provincial, definiendo los aspectos comunes a todas las comarcas, recogiendo una visión 
de futuro del territorio y consensuando un plan de actuación conjunto, mediante una serie de reuniones de reflexión para posteriormente concretar, tomando como base este documento, los aspectos diferenciadores de cada comarca. A consecuencia de ello, todos los aspectos comunes a las zonas rurales de Guipúzcoa y por lo tanto trasladables a la realidad comarcal, se han podido recoger de forma muy similar en los diferentes PDR Comarcales.

En general, tanto en un caso como en el otro, cabe destacar el trabajo de reflexión conjunta y el consenso al que se ha llegado en estas jornadas creando una dinámica de trabajo vinculada, arrojando como resultado, además de las planificaciones realizadas, la asunción de una nueva forma de trabajo, mucho más coordinada y con visión más estratégica.

\section{El diagnóstico difiere en cada territorio en función de la proximidad urbana}

El diagnóstico de situación de las zonas rurales realizado a nivel comarcal se ha basado en una descripción de la realidad de las mismas, en su análisis DAFO y en la sistematización de ésta a través de la identificación de ventajas competitivas y factores críticos. En este caso nos centramos en el diagnóstico del territorio de Vizcaya para luego aportar una visión comparativa de Álava y Guipúzcoa.

En Vizcaya se ha dado preferencia a cinco áreas diferenciadas de las que, sobre cada una de ellas, obtenemos las siguientes conclusiones:

- Turismo. Se llega a la conclusión de que las zonas rurales de Vizcaya poseen una riqueza paisajística y natural y un patrimonio cultural, arquitectónico e histórico de alto valor, con una demanda creciente por parte de los turistas y excursionistas. Además el medio rural ofrece una amplía gama de opciones desde el turismo rural, cultural y de playa hasta otros segmentos en auge como el de naturaleza, el turismo activo, el gastronómico o el turismo de negocios. La cercanía a Bilbao ofrece grandes ventajas como reclamo de turistas, y con grandes posibilidades debido a la saturación que la capital está sufriendo en cuanto a plazas de alojamiento.

En cuanto a la estructuración del turismo en las diferentes comarcas de Vizcaya, se concluye que los factores, en general debilidades, se repiten. Destacar la falta de una visión para abordar su desarrollo, en muchos casos debido a la falta de consideración del turismo como instrumento de revitalización económica y social. En consecuencia la gestión del desarrollo del turismo se 
encuentra poco organizada, no se planifica ni se define el modelo a impulsar y la coordinación entre las administraciones competentes y el sector privado es escasa.

- Demografía, cultura y cohesión comarcal. Población envejecida, con comarcas donde comienzan a detectarse problemas de integración y de identidad por la exclusiva función residencial de las zonas rurales, que deriva en una merma de la vida social del municipio. En las zonas más cercanas a Bilbao la migración de la población de zonas urbanas ha provocado un crecimiento poblacional no sostenido que ha agudizado problemas de integración y de identidad por la tendencia a la exclusiva función residencial de estas zonas rurales. Estos problemas de integración, identidad, pérdida de sentimiento de pertenencia, etc. derivan en una menor participación de la población en la vida social del municipio. Asimismo se apunta falta de visión comarcal y escasa coordinación entre las entidades y organismos que trabajan en el desarrollo socio económico de la comarcas ${ }^{14}$.

- Economía rural y calidad de vida. Se apunta una deficiente oferta en servicios, equipamientos e infraestructuras, ausencia de centros escolares, falta de especialidades en los centros sanitarios, falta de una ambulancia medicalizada y limitaciones del transporte público que dificulta el acceso a los diferentes servicios ${ }^{15}$.

Por otro, se señala el escaso dinamismo empresarial y falta de actividad económica de algunas comarcas. La necesidad de una actividad económica generadora de empleos es palpable en comarcas alejadas de zonas urbanas, vital para la generación de una actividad tractora y dinamismo empresarial. Como grandes oportunidades del medio rural se vislumbra las posibilidades del desarrollo de nuevos yacimientos de empleo relacionados con la gestión del medio físico (guardas, guías de educación ambiental, guías de naturaleza, relacionados con la silvicultura, paisajistas, jardineros...), debido a la constatada demanda cre-

\footnotetext{
${ }^{14}$ La soledad en los espacios rurales, como señala García Rodríguez (1990), se manifiesta en un vacío cultural, en una progresiva pérdida de valores y de identidad, falta de comunicación o en un excesivo tiempo libre. Para evitarlas se ha de impulsar la inserción social, la solidaridad y ayuda mutua, la comunicación y la convivencia. Entre las propuestas sociales que se plantean se cita la dotación de centros de relación y convivencia social, centros de inserción social o, simplemente, recuperación del uso de espacios tradicionales.

15 Responde a la cada vez mayor demanda de la población que vive de forma continuada en el medio rural y que está condicionada por el envejecimiento generalizado de sus habitantes. Es una demanda común de las zonas rurales. Reinoso y Sancho (2002) apuntan a los servicios públicos como los más demandados en la Sierra Norte de Guadalajara, especialmente a la mejora de la asistencia sanitaria y en segundo lugar al incremento del transporte público.
} 
ciente hacia el uso de espacios naturales para el ocio y tiempo libre y para el desarrollo de alternativas lúdicas, como a los relacionados con la atención sociosanitaria y educativa o la prestación de servicios a la propia población.

- Sector primario. El declive en el sector primario es un hecho que se constata en todas las comarcas, con temas comunes que en todas ellas se repiten como la falta de relevo generacional, la baja rentabilidad de las explotaciones en general, la falta de mano de obra, la escasa autoestima y el desprestigio del sector, las mejores expectativas económicas y profesionales en otros sectores, la falta de dinamismo muy relacionado con el escaso nivel formativo, la estructura de las explotaciones agrarias, la escasa diversificación y las dificultades para el acceso al suelo entre otros. La reducida rentabilidad de las explotaciones está unida a la poca diversificación y a la ausencia de aportación de valor añadido de los productos agrarios, por la escasa transformación. Todos estos aspectos hacen ver que el sector primario es un tema clave a afrontar para evitar su desaparición, para lo que cuenta con grandes fortalezas como la tradición y todavía elevada presencia del sector agrícola, en parte profesionalizada y de la riqueza forestal existente. Se apuntan como grandes oportunidades la proximidad de la producción a los potenciales consumidores de productos agroalimentarios, la existencia de un gran número de ferias, el aumento de la demanda de productos de calidad y los circuitos cortos de comercialización

En el caso alavés, las seis comarcas de este territorio, a partir de una serie de reuniones, definen los temas críticos y las ventajas que presenta su medio rural. Los temas críticos coinciden con los de Vizcaya aunque su particularidad estriba en la mayor dependencia de la agricultura de este territorio, junto al alejamiento de centros urbanos de referencia. De manera resumida resaltamos los siguientes: poca conciencia e identidad comarcal; escasa colaboración y coordinación a nivel institucional; éxodo de mujeres y de la población más joven, debido a falta de empleos cualificados y bien remunerados; problemas para el mantenimiento y la renovación del sector primario; sector turístico poco desarrollado; falta de emprendedores en todos los sectores económicos; carencia de regularidad de servicios sanitarios especializados; acusada falta de transporte público entre pueblos rurales y con respecto a la capital; problemática en torno a la vivienda con una serie de preocupaciones como ausencia de integración y convivencia entre población local y nuevos vecinos debido a los modelos urbanísticos.

Las ventajas comparativas del medio rural alavés se sustentan en los pilares básicos de la mayoría de las zonas de profunda impronta rural y espacios de 
montaña como es el gran potencial de turismo, turismo de naturaleza, patrimonial, enológico: vino txakoli e incluso turismo de golf. Al mismo tiempo se cuenta con los elementos para el desarrollo de un sector primario más innovador: recursos naturales, sistemas de riego, experiencia y profesionalidad, etc.

La diferencia principal frente a otras zonas rurales y que le acerca al carácter industrial del País Vasco es la proximidad de determinadas comarcas a grandes vías de comunicación que en la mayoría de los casos han permitido la diversificación económica y el desarrollo de muchas zonas rurales cercanas a núcleos urbanos y zonas industriales para el acceso al empleo ${ }^{16}$. En estas zonas se suceden propuestas relacionadas con actividades de dotación económica y residencial.

Guipúzcoa se caracteriza por ser una provincia urbana, con un medio rural ordenado por un caserío disperso y desarrollado a media altura, entre la ciudad y la montaña. Entre aquellas áreas que se resaltan en su diagnóstico cabe destacar la incidencia de las siguientes:

- Relación entre el conjunto de la sociedad y el medio rural. A pesar de la proximidad del medio urbano, el medio rural se ve necesitado de un reconocimiento y apoyo por parte de éste. Este reconocimiento tiene que hacerse llegar a la administración a la cual se le solicita el desarrollo de una política específica y ajustada a un medio disperso, poco habitado y extenso.

- La situación del sector agrario es otra de las preocupaciones principales. Preocupa la necesidad de proteger suelo rural de la presión urbana, la necesaria incorporación de jóvenes a la agricultura, impulsar el atractivo del empleo agrario frente al industrial, crear servicios específicos dirigidos a mejorar la calidad de vida del agricultor e impulsar nuevo modelos de desarrollo agrario.

- Dotación de servicios a municipios y barrios rurales. A pesar de los avances producidos los últimos años todavía son muchas las necesidades que la población rural tiene en materia dotacional para conseguir cubrir sus necesidades mínimas. Además se plantea la necesidad de cubrir la gestión y el mantenimiento del servicio. Junto a ello, la necesidad de continuar mejorando las infraestructuras básicas, preferentemente agua y caminos.

\footnotetext{
16 Como recogen Cejudo y Navarro (2003) «ni la inversión pública ni la función dinamizadora que deberían cumplir los técnicos de los diferentes GAL se demuestran eficaces para propiciar la generación de desarrollo en los municipios más deprimidos y periféricos de la provincia».
} 
- Necesidad de impulsar el trabajo en común de las ADR, desde la estructura comarcal a sus relaciones con otras comarcas, provincias e incluso a nivel del País Vasco, como modo de obtener sinergias.

El diagnóstico de cada territorio muestra, como era de esperar, una visión común sobre la situación y demandas de los espacios rurales. Entre las preocupaciones destaca la crisis de la agricultura. Apenas hay agricultores, no hay continuidad, las condiciones de trabajo son malas y las posibilidades de atracción de nuevo personal muy reducidas. Preocupa también las deficiencias que se producen en la dotación de servicios básicos; a pesar de los avances dados los pueblos rurales carecen de muchos de los servicios por una normativa que no se adapta a la realidad de estos espacios. Un último aspecto a destacar es la falta de visión de comarca, lo que dificulta el trabajo en pos de un objetivo común, junto a la casi inexistente relación entre comarcas, territorios y administraciones ${ }^{17}$.

Algunos de los puntos fuertes también son comunes, de los que destacamos dos que son básicos a la hora de realizar planteamientos de futuro. En primer lugar, el reconocimiento de la calidad y valoración del medio en el que habitan por el conjunto de la sociedad, como característica capaz de generar riqueza económica (turismo, actividades de la naturaleza, recreo, gastronomía,...) En segundo lugar, la relativa proximidad de una gran zona urbana, elemento que se produce en la mayoría de las comarcas, y que contribuye a aportar empleo e incluso atraer actividades urbanas al medio rural ${ }^{18}$.

Las diferencias principales se establecen en función del grado de proximidad al medio urbano. En algunos casos preocupa la excesiva cercanía, la especulación que provoca sobre el suelo o la construcción de nueva vivienda, con un recién llegado que ni se integra ni le interesa la dinámica social del lugar en el que habita. En otros, interesa convertir el territorio en un espacio atractivo

17 Y más si tenemos en cuenta, como señalan García Rodríguez et al. (2005) que la supuesta novedad de los proyectos Leader no es tanta sino que su auténtica aportación es la posibilidad de crear grupos que den a conocer el éxito de determinadas iniciativas y sus contenidos puedan extenderse con facilidad.

18 García Fernando (1973), en referencia a la sociedad rural española, apunta que las actividades agrícolas no son suficientes para mantener las estructuras comunitarias. Solamente las comunidades con posibilidades de desarrollo extraagrícola, sobre todo turismo, han visto emerger un campo comunitario capaz de hacer frente a la nueva situación. Sin embargo, cabe preguntarse si en el contexto de una sociedad industrializada tales comunidades son capaces realmente de desarrollarse. Desde luego, desde el punto de vista puramente agrario, según el autor, ello no parece posible. 
para las inversiones urbanas, desde llegada de población hasta, y sobre todo, atracción de actividades económicas motoras.

Como podemos observar la aplicación metodológica no varía los resultados obtenidos, similares a los señalados una década antes en los anteriores Planes de desarrollo rural (Alberdi, 2004) y que, salvo la particularidad de la proximidad urbana, apenas difieren de las demandas habituales de otras zonas rurales (Reinoso y Sancho en la serranía de Guadalajara, 2002; Cejudo y Navarro en Granada, 2003; Alario y Baraja en Castilla-León, 2006,...). Lo que la nuevo metodología aporta es simplicidad y puesta en común.

\section{Formulación de la estrategia: hacia un horizonte de futuro con un medio rural con identidad propia}

Además de conocer la situación y saber cuáles son las potencialidades y los obstáculos principales, es importante definir el medio rural al que se quiere llegar. La visión se define como el instrumento que nos acerque a ese escenario que se persigue. Es un trabajo de reflexión y de proyección a futuro, una imagen que combine deseo y posibilidad.

La visión del medio rural del País Vasco se estructura por provincias. Para ello se realiza un ejercicio en el que en primer lugar, cada gerente de la ADR aporta las ideas principales que en su opinión debían ser parte de la visión; en segundo, se realiza una puesta en común y se agrupan las ideas por temas, para posteriormente y por equipos de trabajo, esbozar un primer borrador. A partir de él se consensúa el que va a ser el documento definitivo.

La visión es una puesta en común que parte del modelo de desarrollo que cada comarca quiere aportar a su medio rural y que, trata de buscar consenso para cada provincia. Las visiones aportadas en los distintos territorios se recogen en la tabla 1.

Al igual que el diagnóstico, la visión es en gran medida compartida. En todos los casos se busca mantener un entorno rural vivo y con identidad propia. El futuro pasa por mantener la actividad económica que le ha aportado su carácter actual, la agricultura, una actividad que ha de obtener mayor valor añadido y que ha de diversificarse. Por otro lado, se aspira a conseguir un medio rural en el que la calidad de vida de sus habitantes ha de estar, al menos en cuanto a la dotación de servicios básicos, garantizada. En definitiva, un medio rural sostenible y apoyado en un desarrollo endógeno. 
TABLA 1

HORIZONTE A ALCANZAR EN LAS COMARCAS DEL PAÍS VASCO SEGÚN LAS ADR

«Queremos que el medio rural de Vizcaya sea sostenible, con una economía diversificada, estructurada, coordinada y de calidad, donde el sector primario sea valorado por la labor multifuncional que realiza, con una sociedad participativa e integradora, con identidad propia y autoestima y dotado de todos los servicios y equipamientos o acceso a los mismos, donde el conocimiento y la innovación sean la base del desarrollo y la competitividad.»

«Las zonas rurales de Álava contarán con un sector primario diversificado, basado en criterios de calidad y valor añadido, con un turismo de calidad mediante productos definidos, complementando con otras actividades económicas, manteniendo la población integrada en un entorno rural vivo en el que se garantice el acceso a servicios e infraestructuras, todo ello bajo un marco de desarrollo endógeno y sostenible.»

«En Guipúzcoa queremos un medio rural que garantice el modo de ser y la calidad de la población que los habita, solidario entre sí y con el exterior. Un espacio reconocido por sus peculiaridades, aspecto que se ha de materializar en un marco normativo, económico y dotacional, en el que el la vocación agraria del suelo esté protegida y garantizada. Un medio rural que ofrezca e impulse actividades económicas relacionadas con las características naturales, ambientales y económicas propias de su medio y, entre éstas, especialmente las relacionadas con la actividad agraria, impulsando su valor añadido, su continuidad y un modelo de desarrollo independiente y propio.»

A partir de este esquema general cada visión tiene sus particularidades. Del caso de Guipúzcoa cabe destacar el apunte que realizan sobre la necesidad de que el medio rural apoye su futuro, también el económico, en el desarrollo de actividades relacionadas con sus características ambientales y naturales, un espacio que se ha de proteger explícitamente de la presión del suelo urbano. Álava, por su parte, se apoya en la necesidad de impulsar el turismo como complemento a las actividades agrarias, junto a la incorporación de otras iniciativas, preferentemente de origen urbano, mientras Vizcaya apuesta por una economía diversificada19.

19 Una conclusión a la que llegan muchos estudios relativos a áreas rurales. Según Martín et al. (2005), el objetivo del estudio sobre la montaña abulense es realizar un diagnóstico integral 
La visión coincide con el diagnóstico, en el que la calidad de vida, cohesión social y el desarrollo endógeno eran sus pilares básicos. Las diferencias las vuelve a establecer, al menos en gran medida, la proximidad al medio urbano, más intensa en Guipúzcoa y algunas comarcas Vizcaínas y queda patente en la necesidad de diferenciarse y protegerse de la presión de la ciudad, mientras en buena parte de Álava es observada como una oportunidad de desarrollo.

\section{La planificación se apoya en líneas estratégicas comunes como medio de actuación y de presión}

En la fase de planificación se trata de definir, a partir de la visión, cuáles son los objetivos concretos a perseguir y las líneas de actuación o estrategias que han de marcar el camino a seguir para alcanzar los objetivos. En principio, atendiendo a la metodología planteada, se pretende que sean objetivos alcanzables y líneas de actuación asumibles desde la propia actividad de la ADR.

El proceso de planificación se repite en las tres provincias y, en ambas, se obtienen unos resultados similares. En este caso, tomamos como referencia aquellos datos relativos al territorio Vizcaíno, ordenando las estrategias y los objetivos por ejes. Partiendo de la base de los temas comunes, el proceso de consenso de objetivos y estrategias territoriales se llevó a cabo de la siguiente forma:

- Como primer paso se agruparon los temas comunes en grandes Ejes (o ámbitos de trabajo) y se identificaron entre los planes comarcales aquellos objetivos y estrategias que responden a los Ejes definidos. Con ello se obtuvo un listado previo de ideas que sirvió como base para la posterior definición.

- En una jornada, reunidos en grupos de trabajo, se consensuaron los Ejes y Objetivos principales.

- Finalmente, en otra jornada y teniendo como insumo el listado de estrategias planteado por las comarcas y los objetivos anteriormente definidos, se debatieron y consensuaron las estrategias comunes territoriales.

de las zonas serranas para, a continuación, indicar algunas propuestas de actuación. Se sigue un método de análisis de las principales variables físicas y socioeconómicas del que se deduce que los valores naturales son un recurso para la comarca y que tanto el éxodo demográfico como el abandono de las actividades económicas ha llevado a una desarticulación territorial y a un deterioro en la prestación de servicios. En la actualidad la ganadería y el turismo se perfilan como las dos apuestas de mayor futuro. 
Tras este proceso quedaría por definir los indicadores de los objetivos planteados, y elaborar un Plan de Gestión Anual Territorial, en el que se especifiquen las acciones a llevar a cabo anualmente, con los responsables de cada actuación, fuentes de financiación, indicadores operativos, etc.

El esquema presentado es un desarrollo de los aspectos que a lo largo de este artículo hemos ido recogiendo. Partiendo del nivel comarcal, una vez superado éste, se realiza una puesta a punto y un esfuerzo de concreción importante. Como consecuencia el documento se reduce físicamente y aporta una visión global, que resumimos en la tabla 2 .

TABLA 2

\section{EJES, OBJETIVOS Y ESTRATEGIAS DE DESARROLLO PARA VIZCAYA SEGÚN LAS ADR}

\section{Eje 1. Sector primario}

Objetivo: Mantenimiento de la actividad agrícola.

Estrategias: Impulsar nuevas incorporaciones. Estudiar y promover alternativas que faciliten el acceso al suelo. Facilitar el acceso a las subvenciones en igualdad de condiciones de los agricultores y ganaderos mixtos.

Objetivo: Mejorar la rentabilidad de las explotaciones.

Estrategias: Buscar e impulsar fórmulas que contribuyan al incremento del valor añadido de las producciones agrarias (transformación, comercialización y/o diversificación de la producción) y que repercutan positivamente en el productor. Desarrollar nuevos canales de comercialización.

Objetivo: Mantener la actividad forestal e impulsar su diversificación.

Estrategias: Favorecer la introducción de nuevas especies y usos. Favorecer la segunda transformación.

Objetivos: Concienciar al conjunto de la sociedad sobre la multifuncionalidad del espacio rural y ponerla en valor.

\section{Eje 2. Turismo}

Objetivo: Sensibilizar a la sociedad y las administraciones del potencial del sector como actividad económica.

Objetivo: Conseguir un sector turístico más definido, estructurado y coordinado hacia objetivos comunes y globales.

Estrategias: Impulsar la creación y el desarrollo de las empresas turísticas. Crear productos turísticos en el ámbito de Vizcaya: puesta en valor de los recursos. Desarrollar alternativas para superar la estacionalidad así como nuevas opciones para pasar del turismo de día a viajes con pernoctaciones en el destino. Mejorar la promoción y comercialización. 


\section{Eje 3. Cohesión territorial, cohesión social e identidad}

Objetivo: Impulsar/Mejorar la coordinación entre los distintos organismos a nivel territorial (Bizkaimendi, LandaVizcaya, Landaberri).

Objetivo: Lograr que las actuaciones se desarrollen con visión común.

Objetivo: Búsqueda de soluciones aplicables a todas las comarcas para fortalecer el sentimiento de pertenencia, favorecer la participación de la población, fomentar la integración de los residentes...

Objetivos: Establecer vías de difusión y de participación en la definición de los distintos planes que se desarrollen en la Comarca.

Eje 4. Calidad de vida

Objetivo: Mejorar la calidad de vida de los habitantes de las zonas rurales.

Estrategias: Intermediar ante el departamento de Educación para que en los casos en los que por ley les corresponda se abran los centros educativos pertinentes. Identificar a los posibles usuarios de los servicios sociales y actuar de una manera más proactiva a la hora de ofrecer los mismos. Intermediar ante el departamento de Sanidad para dar respuesta a las necesidades en equipamientos y servicios de salud en el medio rural. Dotar y optimizar de recursos económicos y humanos para mejorar el aprovechamiento y optimización de los centros socioculturales. Garantizar el acceso a banda ancha a todos los vecinos del medio rural. Adecuar y procurar el acceso de la población rural a los servicios y equipamientos.

\section{Eje 5. Actividad Económica}

Objetivo: Impulsar/promover la generación de actividad económica en las zonas rurales.

Objetivo: Aprovechar las oportunidades que ofrece la apuesta existente en torno a la innovación y emprendizaje para desarrollar las zonas rurales.

Los ejes en los que se estructura deja entrever cuales son los aspectos que preocupan a las diferentes Asociaciones de Desarrollo. Agricultura, Calidad de Vida, Diversificación Económica y Turismo, y Cohesión Territorial e Identidad son los que estructuran la fase de planificación de los Planes de Desarrollo de Vizcaya, aspectos todos ellos que ya quedaban patentes desde la fase de diagnóstico ${ }^{20}$.

20 Objetivos compartidos en la mayoría de los programas de desarrollo rural. Como señala Plaza (2005), para el logro de sus objetivos PRODER adoptó una estructura práctica articulada en torno a diversas medidas y acciones, siguiendo, de este modo, un modelo de aplicación muy similar a la iniciativa LEADER, si bien una de las diferencias entre ambos es que en PRODER no se contemplaba la cooperación transnacional que sí existía en LEADER. Las medidas 
En la nueva redacción, el documento tiene la virtud de concretar ejes y también objetivos. Estos últimos tienen que ser un reflejo de lo que queremos alcanzar y, en este sentido, los objetivos que se presentan son concretos, claros y lo suficientemente generalistas como para que se les aporte un tratamiento común.

Las estrategias para alcanzar estos objetivos comienzan a plantear más problemas. Todas las presentadas son estrategias que pueden ser impulsadas desde las ADR pero aquellas cuya materialización depende de ellos son las menos. Las Asociaciones pueden presionar pero la decisión definitiva no está en sus manos y es, generalmente, de corte político. Eso sí, el carácter global que ahora se les aporta a estas estrategias convierte a las ADR en entidades de presión con mucha más fuerza que actuando de manera individualizada.

Las dificultades principales se establecen a la hora de precisar acciones más concretas. En este caso tan sólo se recogen algunas de ellas puesto que muchas de ellas se han de concretar en los planes de actuación que cada comarca ha de abordar anualmente ${ }^{21}$. Aun así, se echa en falta una mayor concreción desde una perspectiva global, aspecto que ayudaría en la dirección de las actuaciones que anualmente se han de materializar.

El carácter global que se aporta a la fase de planificación tiene la virtud de dar fuerza a determinadas demandas y plantear soluciones de manera genérica. Al mismo tiempo, marca las líneas básicas que en cada comarca cada plan de actuación anual ha de definir.

del Programa Operativo de Desarrollo y Diversificación Económica de las Zonas Rurales Objetivo 1 de España aparecidas en la Resolución del 3 de Febrero de 1997 se agruparon en torno a los siguientes ejes financiados, cada uno, por uno $\mathrm{u}$ otro de los fondos estructurales comunitarios que se señalan. El patrimonio, el turismo, las empresas y las propias actividades y potenciales agroforestales se convirtieron en los vértices de referencia de aplicación del programa.

21 «Pero éstas no son las únicas ventajas de diseñar la evaluación de forma simultánea al diseño y aprobación del proyecto; también pueden apuntarse las siguientes: (a) se otorga un marco de referencia adecuado para el Estudio de Base, lo que permite tener un buen punto de comparación para las evaluaciones de mediano plazo, terminal y "ex-post"; (b) se consigue que las actividades de evaluación sean consideradas por los directores y ejecutores del proyecto como parte integrante de la gestión del mismo, y no como un área de fiscalización y control; (c) aclara la ubicación institucional que tendrá el sistema de evaluación, evitando ambigüedades; (d) permite identificar la metodología a utilizar, y planificar la formación del equipo de evaluación; etc.» (Viñas. 1994). 
HOMOGENEIDAD EN LA ELABORACIÓN DE LOS PDR y los Planes de Gestión comarcales.

La consecución de la perspectiva territorial parte de una reflexión comarcal que en algunos casos viene precedida de la elaboración del PDR o en otros, como ocurre en Guipúzcoa, observando que la realidad de cada comarca no es muy diferente una de otra, un documento consensuado, a partir del cual incorporar en cada caso las peculiaridades que se consideren oportunas.

Los PDR resultantes, tanto de un modo como del otro, son documentos muy sintetizados, resumidos, de rápida consulta puesto que carecen de toda la acumulación de información de los anteriores y reducen ampliamente los ejes, objetivos y, sobre todo, las líneas de actuación. Se apuesta por un documento básico, similar al consensuado a nivel territorial, un instrumento guía para la elaboración de los correspondientes Planes Anuales.

En este trabajo no vamos a profundizar en los contenidos de los 18 diferentes PDR que se han efectuado. Sería caer en una excesiva repetición, coincidente por otro lado con las líneas que ya hemos apuntado. Sin embargo, por lo novedoso del planteamiento, vamos a analizar el territorio de Guipúzcoa como muestra del modelo metodológico impulsado ahora en esta Comunidad.

En el caso concreto del Territorio Histórico de Guipúzcoa, una de las mayores diferencias respecto al resto y que ha supuesto uno de los aportes más positivos de todo el proceso de actualización de los PDR en este territorio, ha sido el haber iniciado todo el trabajo de planificación primeramente a nivel provincial, definiendo los aspectos comunes a todas las comarcas, recogiendo una visión de futuro del territorio y consensuando un plan de actuación conjunto, mediante una serie de reuniones de reflexión, para posteriormente concretar, tomando como base este documento, los aspectos diferenciadores de cada comarca.

A consecuencia de ello, todos los aspectos comunes a las zonas rurales de Guipúzcoa y por lo tanto trasladables a la realidad comarcal, se han podido recoger de forma muy similar en los diferentes PDR comarcales. Las peculiaridades se han aportado posteriormente, sobre todo en las presentaciones que cada gerente ha realizado a los miembros de sus respectivas juntas.

Por lo demás, la reflexión provincial se corresponde en su mayor parte con el modelo recogido para Vizcaya. Las inquietudes son análogas aunque difieren algunos objetivos y líneas estratégicas. La preocupación por la situación del sector agrario es similar si bien en Guipúzcoa se pone más relieve en las preocupaciones ambientales y en la necesidad de proteger suelo para la agricultura. Se comparten las propuestas relativas a la mejora de servicios en el 
medio rural, la necesidad de reconocimiento por parte de la administración de las aportaciones de este espacio, la falta de coordinación y la necesidad de movilizar al habitante rural en pos del desarrollo de su barrio o pueblo.

Dentro de ese esquema general cada comarca aporta sus peculiaridades. Del análisis de los diferentes PDR y del primer Plan de Gestión Anual, el correspondiente al 2008, resaltamos en cada caso las peculiaridades siguientes:

- En la Comarca de San Sebastián, área que combina grandes núcleos urbanos con una extensa zona rural calificada en gran parte por figuras de protección del medio natural (Parque Natural de Aiako Harria, Biotopo del Leizaran, Parque Periurbano de Lau Haizeta Red Natura 2000 Jaizkibel,...), se pone especial énfasis en el cuidado y explotación del espacio calificado. La puesta en valor del patrimonio cultural y natural de la zona se convierte en objetivo prioritario puesto que es considerado uno de los motores principales para su desarrollo, origen de la puesta en marcha de actividades ligadas al ocio y turismo. Se pretende atraer visitantes hacia el interior de una comarca que cuenta con focos de turismo costero y centros importantes de población, como la conurbación Donostia-Irun.

- En el Alto y el Bajo Deba, comarcas caracterizadas por su impronta industrial, se pone más énfasis en la necesidad de apoyar al agricultor mixto, que combina la actividad agraria con el trabajo en el medio urbano y que suponen más del $90 \%$ de las explotaciones de esta zona. Aunque no de una manera tan explicita como en San Sebastián, la necesidad de acercar el turismo de la costa al interior es otro de los objetivos prioritarios fundamentándose en la puesta en valor de su medio natural.

- La comarca del valle del Urola incide en la necesidad de diversificación económica de sus pueblos más rurales, si bien las iniciativas planteadas no se salen de la línea marcada para el conjunto provincial (turismo, apoyo al pequeño comercio, energías renovables, patrimonio natural y cultural,...).

- Tolosaldea, la comarca que mayor número de municipios menores de 1000 habitantes presenta en el territorio de Guipúzcoa insiste en las aportaciones de Urola, junto a una apuesta decidida por la transformación y comercialización de productos agrarios.

- Goierri, comarca de fuerte industrialización en sus valles y de serranías en altura recogidas bajo la calificación de parque natural, subraya la necesaria protección del suelo rural, con propuestas dirigidas a su ordenación explicita a partir de las normas subsidiarias. Al mismo tiempo, 
apuestan por promover iniciativas de desarrollo agrario centradas en explotaciones de pequeñas dimensiones, especialmente sistemas de producción que aboguen por la calidad, el medio ambiente y el bienestar animal, como la agricultura ecológica. Resaltan, asimismo, la necesidad de unir medio natural, medio rural y actividad agraria planteando un modelo de desarrollo centrado en estas variables.

Los elementos que para cada comarca hemos subrayado apenas constituyen las únicas diferencias entre unos PDR y otros. Es más, los planes de gestión relativos al 2008 son casi idénticos, con la salvedad de que, dependiendo de cada comarca las localizaciones y denominaciones cambian.

Las diferencias señaladas no son una variable importante y, como se puede observar, responden al planteamiento genérico que, desde la visión, se resaltaba para este territorio. En gran medida, podemos concluir que éstas se producen por la propia dinámica de los técnicos de la ADR, en algunos casos más centrada en la promoción del medio natural, en otros en la transformación y comercialización de productos agrarios, en actividades turísticas o incluso en ordenación territorial.

La situación es similar en todos los casos y las iniciativas planteadas parecidas. Pero no debemos de entender esta homogeneidad como algo negativo sino fruto del desarrollo que van adquiriendo estas entidades, con un grado de diversificación amplio y una capacidad de actuación cada vez mayor, como queda recogido en sus Planes de Gestión. Queda pendiente la asignatura de ampliar su capacidad de influencia y su capacidad para implicar a diferentes instituciones y departamentos.

UNA ALTERNATIVA AL MODELO DE DESARROLlO RURAL ACTUAL.

A MODO DE CONCLUSIÓN

La política de desarrollo rural del País Vasco se ha visto progresivamente impulsada por los diferentes programas que desde la Unión Europea se iban poniendo en marcha en materia de desarrollo rural. Su carácter parcial (amplias zonas quedaron fuera de su aplicación) y la reducción de los fondos comunitarios aportados hacían necesario plantear un proceso de actuación diferente en esta región, fundamentado en una participación activa y coordinada en materia de desarrollo rural de las diferentes instituciones con las que cuenta la región y que inicia su andadura con la aprobación de la Ley de Desarrollo Rural en 1998. 
Tras una década se plantea una revisión de los resultados obtenidos observándose deficiencias importantes en el modelo elegido. En general, éste se mantiene en su estructura organizativa, es decir, coordinación e implicación administrativa y programas de desarrollo y asociaciones comarcales como medio de captar necesidades, plantear y ejecutar actuaciones. Sin embargo, cambia el modo de realizar los programas de desarrollo, centrados en la necesidad de sintetizar objetivos y aportar una visión común de la problemática de las diferentes comarcas. También difiere el modo de actuar, más realista y consecuente con las posibilidades de financiación a las que pueden acceder las Asociaciones.

En términos generales, llegamos a la conclusión de que tras las variadas décadas dedicadas a la promoción del medio rural se ha impuesto un modelo de actuación muy pragmático, preocupado por no generar falsas expectativas entre la población rural y primando la gestión ágil y realista. Ello supone una alteración del modo de actuación tradicional que se concreta en, al menos, tres estadios diferentes:

- La metodología utilizada en la redacción de los Planes de Desarrollo Rural mantiene la estructura tradicional pero se apuesta ahora por vaciarla de mucho de su contenido habitual. No se busca la participación de todos los agentes y personas implicadas, las reuniones se reducen, los aspectos descriptivos se suprimen y el documento final pierde volumen. Sin embargo se gana en operatividad y direccionalidad.

- La problemática y demandas rurales no difieren demasiado de unas zonas a otras. La metodología muestra matices locales en una realidad global, apreciaciones que en el País Vasco parecen estar relacionadas con la mayor o menor proximidad al medio urbano.

- La implicación de los agentes que pueden intervenir en el desarrollo rural es clave, especialmente de la administración pública, a la que se le solicita una política específica para el medio rural. Para ello es necesario que las reivindicaciones de las comarcas y territorios vascos sean comunes y conseguir que sean definidas líneas de actuación aplicables a todo el territorio.

- Los objetivos son compartidos, es decir, conseguir un medio rural vivo, independiente y diferenciado en una gran región urbana, como es el conjunto del País Vasco.

La evolución experimentada en las políticas de desarrollo rural del País Vasco es, cuanto menos, peculiar. Por lo general, las diferentes iniciativas de desarrollo rural se sustentan fundamentalmente en las posibilidades presu- 
puestas que las políticas europeas, con la colaboración estatal o regional, han puesto en marcha. Han tenido unos resultados destacados, consecuencia en gran medida de la importante dotación económica que se ha aportado. Pero, ¿que va a ocurrir una vez que las aportaciones comunitarias se reduzcan o desaparezcan? ¿Se van a mantener las estructuras de dinamización local actuales?

El modelo de desarrollo rural puesto en marcha en el País Vasco puede ser una iniciativa a imitar por otros territorios, una vez que las aportaciones comunitarias se reduzcan y tenga que ser la iniciativa local o regional la que haya de asumir la responsabilidad de impulsar el progreso de las zonas rurales. Aun así, pocas regiones tienen la peculiaridad de contar con un medio rural de extensión reducida, próximo al espacio urbano, altamente valorado por su población y que, a la postre, justifica socialmente un alto gasto en políticas de intervención de apoyo al medio rural.

El modo de proceder, sin embargo, aún ha de mostrar su valía. La estructura, apoyada en la elaboración de planes de desarrollo rural locales y en entes dinamizadores de gran experiencia parece la adecuada, la coordinación interadministrativa e interderpartamental es necesaria y la proximidad del medio urbano un recurso para la dinamización de las economías locales. El escaso peso de lo rural en la economía vasca, que hace de este espacio un medio débil, ideal para el desarrollo de iniciativas exógenas, que poco le aportan, junto a la escasa voluntad política de intervención en materia dotacional y de ordenación que ha habido hasta el momento son asignaturas que la estructura de desarrollo rural ha de abordar.

Recibido: 18/09/2008

Aceptado: 29/04/2010

\section{BibliografíA}

Alberdi, J. C. (2004): Colaboración interinstitucional en el desarrollo rural: aciertos y fracasos de la Ley de Desarrollo Rural del País Vasco. Boletín de la Asociación de Geógrafos Españoles, 7-30, nº 33.

Bristow, G., Cowwell, R. y Marsden, T. (2001): Tensions, limits and potentials. Evaluating rural development policies in Scotland. European Urban and Regional Studies, 8: 235-252.

Casley, D. J. y Kumar, K. (1990): Recopilación, análisis y uso de los datos de seguimiento y evaluación. Madrid. Ediciones Mundi-Prensa/Banco Mundial, 179 pp. 
Cejudo García, E. y Navarro Valverde, F. N. (2003): El reparto territorial de los programas de desarrollo rural. El caso de la provincia de Granada. Anales de Geografía de la Universidad Complutense 2003, 23, 131-162.

Espacia, J. y Noguera, J. (2000): Aproximación teórico-metodológica a la cultura evaluativa y la evaluación de programas de desarrollo rural. Cuadernos de Geografía Universidad de Valencia. 67-68: 77-101.

García Ferrando, M. (1973): El futuro de la pequeña comunidad rural y el desarrollo planificado. Revista de Estudios Agrosociales, n. 85, 1973, pp. 93-119.

García Rodríguez J. L., Febles Ramírez, M. F. y Zapato Hernández, V. M. (2005): La iniciativa comunitaria Leader en España. Boletín de la Asociación de Geógrafos Españoles, 39: 361-398.

López, A. et al. (2003): El desarrollo local en la sociedad red. La villa de Graus, un diagnóstico interactivo. Zainak. Cuadernos de Antropología-etnografía, 24: 739-760.

Martín Martín, E., López Pastor, A.T. y Del Barrio Aliste, J. M. (2001): Programas de desarrollo, actividades innovadoras y empleo. Lecciones, estrategias y recomendaciones para el desarrollo rural de Castilla y León. Revista de investigación económica y social de Castilla y León, $\mathrm{n}^{\circ}$ 4, 2001, pp. 9-117.

Mendikoi (1998): Plan de desarrollo rural de las comarcas de Montaña Alavesa, Valles Alaveses, Rioja Alavesa, Estribaciones del Gorbea, Cantábrica Alavesa, Llanada Alavesa, Enkarterrialde, Arratia Nervión, Tolosaldea-Urola Kosta. Departemento de Agricultura del Gobierno vasco. Vitoria. Inédito (formato CD).

Mendikoi (2002): Plan de desarrollo rural de las comarcas de Durangaldea, Uribe, Busturialdea, Lea-Artibai, Debabarrena, Donostialdea, Goierri, Debagoiena. Departemento de Agricultura del Gobierno vasco. Vitoria. Inédito (formato CD).

Mendikoi (2003): Ley de Desarrollo Rural y Programas de Desarrollo Rural. Jornadas de reflexión 14, 15, 18 y 24 de julio de 2003. Departamento de Agricultura del Gobierno vasco. Vitoria. Inédito, $289 \mathrm{pp}$.

Moreno Benito, M. P. (1996): "Disposiciones planteadas para el desarrollo rural en las zonas de montaña: la provincia de Cuenca". VIII Coloquio de Geografía Rural. Jaca, pp. 265-276.

Murray, R. (2002): "Citizens Control of Evaluations: Formulating and Assessing Alternatives". Evaluation, vol. 8, n 1, pp. 81-100.

Patton, M. Q. (2002): "A Vision of Evaluation that Strengthens Democracy". Evaluation, vol. 8, no 1, pp. 125-139.

Plaza Gutiérrez, J. I. (2005): Desarrollodiversificación en las zonas rurales de España: el programa Proder. Boletín de la A.G.E, no 39, pp. 399-422.

Reinoso, D. y Sancho, J. (2002): "Equipamientos y servicios en la Sierra Norte de Guadalajara: un diagnóstico basado en la participación social". X Coloquio de Geografía Rural. Santander, pp. 772-779.

Viñas, V. (2004): Evaluación cualitativa de programas de desarrollo regional en zonas rurales. Revista de estudios regionales, 71: 13-36. 
Wojan, T. y Rupasingha, A. (2001): Crisis as opportunity: local context, adaptive agents and the possibilities of rural development. Regional Studies, 35: 414-152.

Zapatero Zapatero, J. (1998): Instrumentos específicos de desarrollo rural: la Iniciativa Comunitaria LEADER y el Programa Operativo PRODER. Polígonos: Revista de geografía, no 8, pp. 21-38.

\section{RESUMEN}

En el año 1998 el País Vasco pone en marcha un modelo de desarrollo rural fundamentado en la colaboración entre administraciones locales y regionales frente a la dependencia de las subvenciones comunitarias. Con objeto de la necesaria renovación de los Planes Comarcales se produce una reflexión sobre los errores cometidos y se plantean diferentes alternativas que quedan recogidas en unas propuestas metodológicas que van a primar planteamientos inductivos, prácticos y ejecutables y un modo de funcionar que permite llegar con facilidad de la propuesta local a la puesta en común regional. Como resultado obtenemos una interesante propuesta de desarrollo, a tener en cuenta, en un momento en el que muchas Comunidades Autónomas han de reorganizar sus redes de desarrollo rural ante la anunciada reducción de aportaciones comunitarias.

Palabras Clave: planificación; desarrollo rural; País Vasco; medio rural; grupos de acción local.

\section{ABSTRACT}

In 1998 the Basque Country starts up a model of rural development based on the collaboration between local and regional administrations and to limit the dependency of the Europe communitarian subventions. With the beginning of the renovation of the local development plans a reflection based on the committed errors. Methodological systems there are propose prioritizing inductive, feasible and practical expositions, and a functional model to pass to the local level from the regional level. As result we obtain an interesting proposal of development to in a while consider in which many Communities have to reorganize their networks of rural development before the announced reduction of communitarian contributions.

KEY WORDS: planning; rural development; Basque Country; rural area; local action groups.

\section{RÉSUMÉ}

Durant l'année 1998 le Pays Basque met en marche un modèle de développement rural fondé la collaboration entre des administrations locales et régionales face à la dépendance des subventions communautaires. Dans le but de la rénovation nécessaire des Plans Régionaux se produit une réflexion sur les erreurs commises et on pose différentes alternatives qui sont reprises dans des propositions méthodologiques qui 
vont favoriser des approches inductives, pratiques et possibles et une manière de fonctionner qui permet d'arriver avec facilité de la proposition locale à la mise en commun régionale. Comme résultat nous obtenons une intéressante proposition de développement à tenir compte à un moment où beaucoup de Communautés Autonomes doivent réorganiser leurs réseaux de développement rural devant l'annoncée réduction de contributions communautaires.

MoTS CLÉs: planification; développement rural; le Pays Basque; moyen rural; groupes d'action locale. 